Rocheleau, C.A., Webster, G.D., Bryan, A., \& Frazier, J. (2004). Moderators of the relationship between exercise and mood changes: Gender, exertion level, and workout duration. Psychology \& Health, 19(4): 491-506. (Aug 2004) Published by Taylor \& Francis (ISSN: 1476-8321). DOI: 10.1080/08870440310001613509

\title{
Moderators of the Relationship between Exercise and Mood Changes: Gender, Exertion Level, and Workout Duration
}

Courtney A. Rocheleau, Gregory D. Webster, Angela Bryan, and Jacquelyn Frazier

\begin{abstract}
Although the role of exercise in improving mood is well established, less is known about moderators of this effect, particularly outside of the laboratory. The current study examined the effect of gender, type of exercise, level of exertion, and duration of workout on the exercisemood relationship in a naturalistic setting. One hundred and thirty five participants (64 females) either engaged in weight training $(n=52)$ or cardiovascular $(n=83)$ exercise. Pre- and postexercise assessments of mood were collected and changes in two types of mood, negative mood and exhaustion, were examined. Overall, participants reported improved mood after exercise in both domains. Workout duration and level of exertion significantly moderated the relationship of exercise to mood improvement (i.e., an increase in either corresponded to greater improved mood, ps $<0.05)$. Overall, women showed more improved mood than men in the domain of exhaustion $(\mathrm{p}=0.001)$.
\end{abstract}




\section{INTRODUCTION}

Evidence regarding the health benefits of exercise is abundant and unequivocal. Despite this general knowledge that exercise is beneficial, most people do not get the recommended minimum amount of exercise of 30 min a day, five or more days a week (Centers for Disease Control and Prevention [CDC], 2001). As of 1998, only $25 \%$ of Americans achieved the recommended amount of exercise per week, and almost $29 \%$ reported no physical activity at all (CDC, 2001).

Regular exercise decreases risk for several diseases, including coronary heart disease, hypertension, certain types of cancer, obesity, osteoporosis, type 2 diabetes, and reduces the overall risk of dying prematurely (Bouchard et al., 1994; Blair et al., 1996; Carpenter et al., 1999; CDC, 2001). These potential benefits, coupled with low rates of exercise, led the U.S. Department of Health and Human Services (2000) to cite exercise promotion as a top priority for the 21st century in the Healthy People 2010 Report.

A limitation of previous exercise promotion programs is that, although participants often adopt an exercise routine as a result of these programs, they do not tend to maintain this behavior after the intervention ends (Sherwood and Jeffery, 2000). In one study, of those who adopted an exercise program of moderate or vigorous activity, 30 and 50\% (respectively), lapsed after a few months (Sallis et al., 1986). Part of the reason that people are not successful in maintaining regular exercise may be that they do not perceive sufficient immediate benefits of exercising. Many of the benefits of exercise are not discernable (e.g., the absence of a disease), and even those that are observable (e.g., muscle tone, weight loss) take time to accrue to the point where they are recognizable. If exercise is not initially rewarding, it is unlikely that people will continue.

One immediate benefit of exercise is psychological. In general, positive mood tends to be enhanced after exercise (see Yueng, 1996 for review). The relationship between chronic exercise and mood enhancement is well established (Gauvin and Spence, 1996), so much so that exercise has been used widely as a treatment for depression and anxiety (e.g., Salmon, 2001; Mather et al., 2002). Research has also suggested that mood benefits are apparent after a single bout of exercise (Steptoe et al., 1993; Pronk et al., 1994).

It may be possible for interventions designed to increase exercise behavior to capitalize on this immediate psychological benefit in order to help people adopt and maintain regular exercise routines. Before this is feasible, however, basic research is required to understand the necessary parameters (e.g., minimum duration of workout, type of workout) for successfully inducing the largest increases in positive mood and decreases in negative mood after exercise. Further, it is important to elucidate specific characteristics of the individual (e.g., age, gender) or the physical activity (e.g., perceived exertion, workout duration) that may moderate the exercise-mood relationship in order to design maximally effective exercise interventions.

Several potential moderators have been examined in previous studies. A recent study by Hansen et al. (2001), for example, examined the effect of workout duration on the exercise-mood relationship. They found that mood benefits were observed with as little as 10 min of exercise, and that increasing workout duration to 30 min resulted in only 
minimal additional mood improvement. Level of exertion also moderates the exercisemood relationship (Farrell et al., 1987; Felts et al., 1988; Zervas et al., 1993), though the specific effect of exertion is not unequivocal across studies. In most studies, exertion has had a linear effect on mood, such that greater exertion leads to more mood change (Farrell et al., 1987; Felts et al.,1988). However, other researchers have observed a quadratic effect of exertion, such that a moderate level of exertion led to more mood change than either low or high exertion (Zervas et al., 1993).

Type of exercise may also affect mood enhancement, though again the evidence is not unequivocal regarding which type of exercises result in increased positive mood. The majority of work on the exercise-mood relationship supports the contention that cardiovascular exercise leads to mood improvement (see Yueng, 1996 for review). On the other hand, while some researchers have found weight training to be equally effective in enhancing mood (Dyer and Crouch, 1988; McGowan et al., 1991), others have shown that only cardiovascular activity is effective (Raglin et al., 1993).

The role of gender as a moderating factor in the exercise-mood relationship has also been examined. On the whole, the exercise-mood relationship has typically been stronger among women than men (e.g., Hansen et al., 1997), but occasionally no gender difference is observed in improved mood (Roth, 1989). One reason that women may exhibit greater mood benefits from exercise is that women tend to report more pre-exercise levels of depression and anxiety than men (e.g., Merns, 1995); individuals who report a worse mood before exercise generally derive the most subsequent benefit (cf., Yeung, 1996). In addition, women tend to report mood enhancement as a motivation for exercising more often than do men (e.g., Tiggeman and Williamson, 2000; Twamley, 2000). Perhaps this expectation leads to greater mood enhancement after exercise. Many previous studies have examined the exercisemood relationship in only men or women (e.g., Steptoe et al., 1993; Lane et al., 2002). It is desirable to include both men and women in the context of a single study so that gender differences can be examined via direct comparison.

One limitation of previous research is that moderators of the exercise-mood relationship have been studied almost exclusively in laboratory settings. In such studies, participants are typically instructed to walk on a treadmill, oxygen consumption is measured and heart rate is continuously monitored, and the incline of the treadmill is adjusted to maintain the desired exertion level. As in any other area of research, controlled laboratory studies with random assignment have higher internal validity than naturalistic designs. Indeed, all of our hypotheses in the current study are based upon findings from laboratory investigations. However, these conditions differ dramatically from the settings in which exercise typically takes place; exercise does not typically involve an experimenter assigning you to a particular exercise (usually cycle ergometer), a particular intensity, and a particular duration. The fact that laboratory exercise differs so radically from exercise in the natural environment may threaten the ecological or external validity of such studies. It is possible that these methods may even disrupt the psychological or physiological mechanisms involved in increasing mood as a result of exercise. It is therefore important to examine this relationship under the conditions in which exercise naturally occurs to fully understand the phenomenon. It is naturalistic studies such as our own that maximize external validity, and thus a combination of studies such as ours as well as controlled laboratory investigations is crucial in order to maximize both internal and external validity. In sum, while laboratory studies offer a greater degree of control and allow for 
experimental manipulations of some moderating variables, they should be conducted in concert with more naturalistic studies in a "full cycle" approach to determine whether the moderating effects occur in the same way in actual exercise settings.

The current study was designed to better identify the moderating effects of gender, activity type, duration of workout, and level of exertion during workout, on the relationship between exercise and mood inflation. Consistent with prior research, we hypothesized that women would show more mood improvement than men, perhaps due to a less positive mood prior to exercise. We also expected that cardiovascular exercise would result in greater mood enhancement than weight training. Among potential mechanisms for the relationship between exercise and mood is the endorphin hypothesis, which posits that exercise leads to release of natural mood-enhancing chemicals in the brain. We predicted that, given such a mechanism, it would be necessary to elevate and maintain a moderate to high heart rate to lead to enhancement in mood. We further predicted that duration of workout and level of exertion would exhibit positive linear relationships with mood, such that increasing duration and exertion would result in greater mood enhancement. The literature generally supports that the relationship between exertion and mood improves linearly until individuals reach a relatively high level of exertion (80-85\% of maximum heart rate; cf., Yeung, 1996). It is only when this extremely high level of exertion is included that a curvilinear relationship appears. We did not expect that the majority of recreational exercisers in our study would be pushing themselves to the exertion level required to see a curvilinear effect. Thus, we predicted that our findings would be similar to most past studies in finding a linear relationship between exertion and mood improvement.

\section{METHODS}

Participants were recruited while preparing to engage in either cardiovascular (e.g., running, aerobics, bicycling) or weight training (lifting free weights or using weight machines) exercise at a university student recreation center. In order to examine gender differences, equal number of males and females were recruited in each type of exercise. To do so, males were over-recruited in the cardiovascular condition and females were over-recruited in the weight training condition. The majority of participants (84\%) were white. Participation was voluntary and anonymous.

\section{Procedures and Materials}

A table was set up at the campus recreation facility advertising the opportunity for a granola bar in exchange for being in a short study related to exercise. Participants were told that the study was completely anonymous (they were identified by subject number only) and that they would be asked to complete one measure prior to their chosen workout and one measure after their workout. The pretest measure consisted of questions about the participant's age and gender. In addition, the Profile of Mood States (POMS; McNair et al., 1971) was used to measure mood. The POMS has been used extensively with similar populations and in research on the exercisemood relationship (see Yueng, 1996). The POMS is generally subdivided into six mood subscales, including: (a) Tension (k=9, e.g., "tense", "jittery"); (b) Depression (k=12, e.g., "sad", "dejected"); (c) Anger (k=6, e.g., "angry", "grouchy"); (d) Vigor 
(k=9, e.g., "lively", "vigorous"); (e) Fatigue (k=4, e.g., "tired", "fatigued");

(f) Confusion (k=14, e.g., "confused", "mixed-up"); and some investigations find a seventh subscale of Friendliness (k=6, e.g., "friendly", "kindly"), (McNair et al., 1971, p. 7). All subscales included some reverse-scored items. The POMS takes about 3-4 min to complete. Participants were instructed to rate their moods "right now," in relation to the 72 adjectives comprising the POMS. Responses were recorded on a 4-point continuum from 0 (much unlike this) to 3 (much like this).

Immediately after completing their chosen workout, participants returned to the same table and completed the post-exercise survey. In this measure, participants again completed the POMS, and were asked to report the type and duration of their workout, and their Rating of Perceived Exertion (RPE), which ranged from 0 (nothing at all) to 10 (maximal exertion). Upon completion, participants were thanked and offered a granola bar as compensation for their time. All procedures were reviewed and approved by a university-level internal review board.

\section{RESULTS}

\section{Sample Characteristics}

Of the 138 participants, one did not report an RPE score, one had an unusually long workout duration of $150 \mathrm{~min}$, and one produced an outlying residual in a subsequent analysis. These three cases were excluded from the current data. Of the 135 remaining participants, 64 (47\%) were female and 71 (53\%) were male. Eighty-three (61\%) of the participants took part in cardiovascular exercise, whereas $52(39 \%)$ lifted weights. By design, we recruited equal number of males and females into each activity type, so that gender did not differ by activity type. The average age of participants was 22.95 years $(S D=6.22$, range $=16$ to 58$)$. Participants spent an average of $46.75 \mathrm{~min}$ ( $S D=24.91$, range $=10$ to $120 \mathrm{~min}$ ) exercising and reported a mean RPE score of $6.44(\mathrm{SD}=1.69$, range $=2$ to 10$)$.

\section{Data Reduction}

The primary objective of this study was to examine changes in mood due to exercise. To this end, we used the POMS scores immediately following exercise as the dependent variable, controlling for pre-exercise POMS scores as a covariate. Rather than investigating the effects of exercise on each of the seven POMS subscales separately, leading to a cumbersome presentation of results, we chose to condense the dependent variables into factors. First, each post-exercise POMS subscale was regressed onto its respective pre-exercise POMS subscale. The resulting set of seven unstandardized residuals was then submitted to a principal components analysis with orthogonal rotation. This procedure revealed two factors. The first factor (Eigen value $=3.50$, variance Explained=50\%), which we called negative mood, consisted of the POMS subscale residuals of anger, depression, tension, friendliness (negative loading), and confusion (see Table I). The second factor (Eigen value=1.01, variance explained=14\%), which which we named exhaustion, included both fatigue and vigor (negative loading). Factor scales were created by reverse coding items in the friendliness subscale for the negative mood factor and vigor for the exhaustion factor. 
TABLE I Principal components analysis of post-exercise POMS subscales ${ }^{\mathrm{a}}$

\begin{tabular}{lcc}
\hline POMS Subscale & \multicolumn{2}{c}{ POMS subscale factors } \\
\cline { 2 - 3 } & Negative mood & Exhaustion \\
\hline Depression & $\mathbf{0 . 8 7}$ & 0.26 \\
Tension & $\mathbf{0 . 7 2}$ & 0.25 \\
Friendliness & $-\mathbf{0 . 7 0 ^ { \mathrm { b } }}$ & -0.03 \\
Confusion & $\mathbf{0 . 6 8}$ & 0.46 \\
Anger & $\mathbf{0 . 6 8}$ & 0.00 \\
Fatigue & -0.02 & $\mathbf{0 . 9 3}$ \\
Vigor & -0.52 & $\mathbf{- 0 . 6 0 ^ { \mathrm { b } }}$ \\
\hline
\end{tabular}

Note: Factor loadings $>0.60$ are indicated in boldface. ${ }^{\text {The }}$ Thependent variables were the residuals from regressing each post-exercise POMS subscale on its respective pre-exercise POMS subscale. Orthogonal rotation was used; "Items from these subscales were reverse-coded prior to calculating factor scale means.

To check for individual differences in pre-exercise negative mood and exhaustion, each of these dependent variables was regressed onto the effects of exercise type, participant gender, workout duration, RPE, and all the two-way interactions of these variables. This set of predictors explained a significant amount of variation in neither pre-exercise negative mood nor exhaustion, $\mathrm{Fs}(10,124)<1$, ps $>0.40$, and no single predictor was related ( $p s>0.20$ ), except for participant gender, which was associated with pre-exercise exhaustion, $b=-0.11$, beta $=-0.22, t(124)=2.16, p=0.03, p^{2}=0.04$, such that women reported more exhaustion at the start of the study $(M=1.37)$ than men $(M=1.15)$.

Workout duration positively covaried with RPE, $r(133)=0.24, p<0.01$. We also examined the extent to which each of these variables was influenced by pre-exercise mood as well as participants' gender and type of exercise. When workout duration was regressed onto type of exercise, gender, their interaction, and pre-exercise negative mood and exhaustion, reliable differences emerged, $F(5,129)^{1 / 412.69}, p<0.001$, $\mathrm{R} 2 \frac{1}{4} 0.33$. There was a significant main effect of exercise type, such that people who chose weight training exercised longer $(M=61.81 \mathrm{~min})$ than those who chose a cardiovascular workout $(\mathrm{M}=44.74 \mathrm{~min}), \mathrm{b}=8.54$, beta $=0.33, \mathrm{t}(129)=4.62$, $\mathrm{p}<0.001, \mathrm{pr}^{2}=0.14$. This exercise type difference was qualified by an interaction with participant gender, $b=11.10$, beta $=0.45, t(129)=5.85, p<0.001, p^{2}=0.21$, such that the exercise type difference in workout duration was observed among men (Mweight training $=73.08 \mathrm{~min}$, Mcardiovascular=33.80 $\mathrm{min}$ ), but not among women (Mweight training=50.54 $\mathrm{min}$, Mcardiovascular=55.68 $\mathrm{min}$ ). This may be due to the fact that men tend to weight lift in groups more often than women. The time spent in spotting group members and waiting until all group members have completed an exercise before moving on to the next exercise associated with men's weight lifting workouts may account for this duration difference.

When we regressed RPE scores onto the same set of predictors, these variables did not explain a significant amount of RPE variance, $F(5,129)<1, p>0.70$, nor did any single predictor emerge ( $p s>0.18$ ). When used as independent variables, pre-exercise POMS scores, duration of workout, and RPE were mean-deviated to aid in the interpretation of the resulting regression coefficients. 


\section{NEGATIVE MOOD}

The post-exercise negative mood scores were regressed onto exercise type (cardiovascular vs. weight training), participant gender, workout duration (min), RPE, and all possible two-way interactions, controlling for pre-exercise negative mood scores. The results of this multiple regression are displayed in Table II. Not surprisingly, pre- and post-exercise negative mood scores were positively correlated. After accounting for this covariation, the other variables in the model explained a significant amount of the variance in the post-exercise negative mood scores, $\Delta \mathrm{R} 2=0.19, \mathrm{~F}(10,123)=$ 3.89, $\mathrm{p}<0.001$, a moderate-to-large effect size (Cohen, 1988).

Participant gender was a reliable predictor of post-exercise mood, with women reporting lower post-exercise negative mood $(M=0.57)$ than men $(M=0.71)$.

However, this gender difference was qualified by an interaction with type of exercise, such that, among those lifting weights, women had lower post-exercise negative moods $(M=0.52)$ than men $(M=0.80)$, whereas no gender difference emerged for those who partook in cardiovascular exercise (Mwomen=0.63, Mmen=0.62).

Workout duration was also related to post-exercise negative mood, such that, for every half hour participants exercised, their post-exercise negative moods decreased by 0.16 raw units or 0.51 standardized units. This effect was qualified by an interaction with RPE, such that, at the highest RPE level, workout duration had no effect on negative mood, whereas, at the lowest RPE level, increased workout duration substantially decreased post-exercise negative mood (Fig. 1).

TABLE II Post-exercise negative mood as a function of individual differences: regression results

\begin{tabular}{lcccc}
\hline Variable & $b$ & $\beta$ & $t$ & $p^{2}$ \\
\hline Intercept & 0.640 & - & $25.21^{* * *}$ & 0.84 \\
Negative mood before exercise & 0.351 & 0.42 & $5.81^{* * *}$ & 0.22 \\
Exercise type & 0.015 & 0.05 & 0.59 & 0.00 \\
Participant gender & 0.066 & 0.22 & $2.64^{* *}$ & 0.05 \\
Workout duration (min) & -0.005 & -0.42 & $3.83^{* * *}$ & 0.11 \\
Rating of Perceived Exertion (RPE) & -0.005 & -0.03 & 0.36 & 0.00 \\
Exercise type $\times$ participant gender & 0.074 & 0.24 & $2.90^{* *}$ & 0.06 \\
Exercise type $\times$ workout duration & 0.001 & 0.04 & 0.50 & 0.00 \\
Exercise type $\times$ RPE & -0.043 & -0.24 & $2.83^{* *}$ & 0.06 \\
Participant gender $\times$ workout duration & 0.002 & 0.17 & 1.56 & 0.02 \\
Participant gender $\times$ RPE & 0.013 & 0.07 & 0.91 & 0.01 \\
Workout duration $\times$ RPE & 0.001 & 0.17 & $2.14^{*}$ & 0.04 \\
\hline
\end{tabular}

${ }^{*} p<0.05 ;{ }^{* *} p<0.01 ;{ }^{* * *} p<0.001$. 


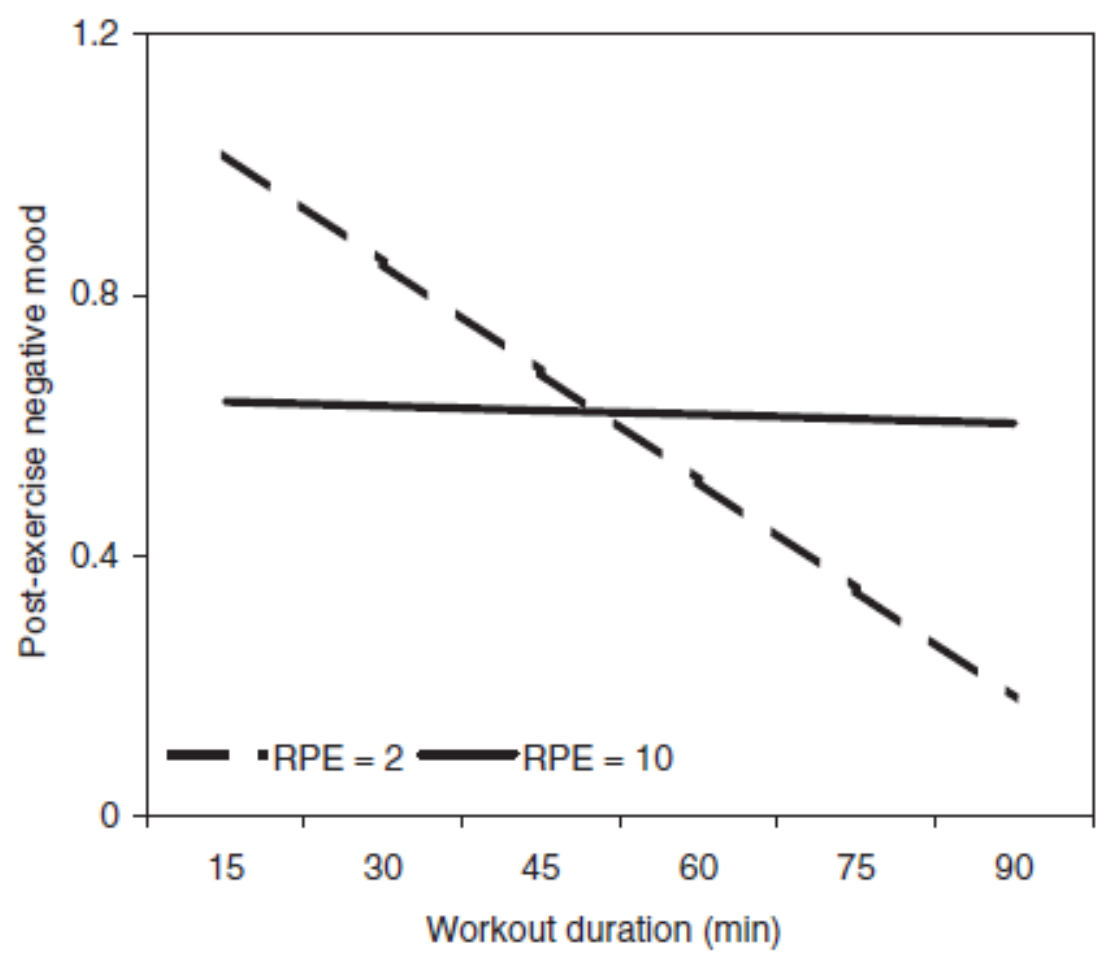

Figure 1 Post-exercise negative mood as a function of workout duration (min) and Rating of Perceived Exertion (RPE).

A reliable exercise type $x$ RPE interaction also emerged, such that the effect of increased RPE on post-exercise negative mood was beneficial for weight trainers (i.e., negative mood reduction), but detrimental for people who engaged in cardiovascular exercise (Fig. 2). 


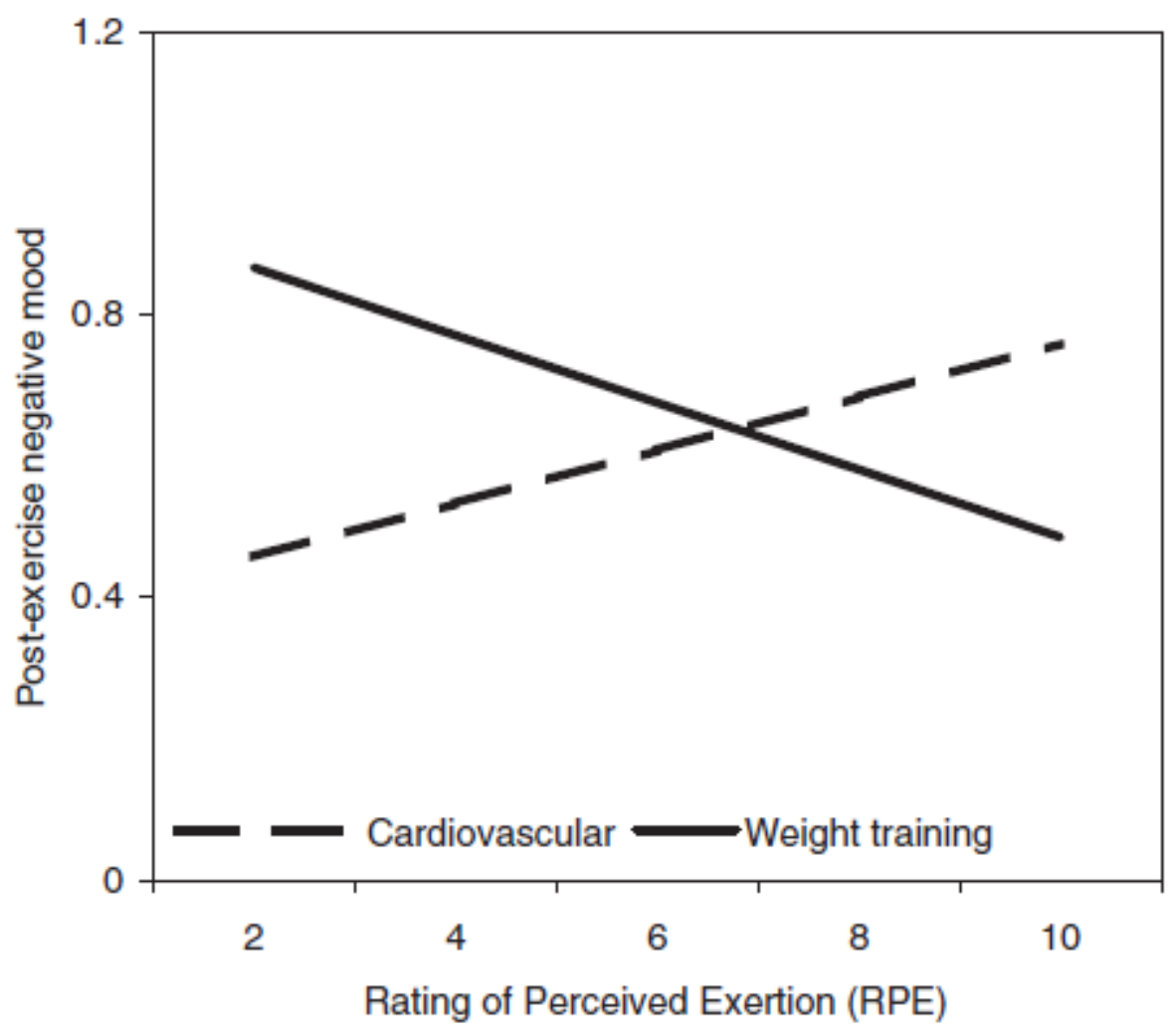

Figure 2 Post-exercise negative mood as a function of exercise type and Rating of Perceived Exertion (RPE).

\section{Exhaustion}

The post-exercise exhaustion scores were regressed onto the same variables as in the negative mood model, controlling for pre-exercise exhaustion scores. The results of this multiple regression are displayed in Table III. Interestingly, pre- and post-exercise exhaustion scores were not reliably correlated. Controlling for pre-exercise exhaustion, the other variables in the model explained a significant amount of the variance in the post-exercise exhaustion scores, $\Delta R 2=0.24, F(10,123)=3.91, p<0.001$, a large effect size (Cohen, 1988). 
TABLE III Post-exercise exhaustion as a function of individual differences: regression results

\begin{tabular}{lcccc}
\hline Variable & $b$ & $\beta$ & $t$ & pr $^{2}$ \\
\hline Intercept & 1.133 & - & $23.25^{* * *}$ & 0.81 \\
Exhaustion mood before exercise & 0.095 & 0.09 & 1.12 & 0.01 \\
Exercise type & 0.034 & 0.06 & 0.70 & 0.00 \\
Participant gender & 0.149 & 0.28 & $3.04^{* *}$ & 0.07 \\
Workout duration (min) & -0.006 & -0.29 & $2.40^{*}$ & 0.04 \\
Rating of Perceived Exertion (RPE) & 0.020 & 0.07 & 0.77 & 0.00 \\
Exercise type $\times$ participant gender & -0.044 & -0.08 & 0.90 & 0.01 \\
Exercise type $\times$ workout duration & -0.002 & -0.10 & 1.07 & 0.01 \\
Exercise type $\times$ RPE & 0.010 & 0.03 & 0.35 & 0.00 \\
Participant gender $\times$ workout duration & 0.008 & 0.36 & $2.90^{* *}$ & 0.06 \\
Participant gender $\times$ RPE & 0.064 & 0.20 & $2.40^{*}$ & 0.04 \\
Workout duration $\times$ RPE & -0.001 & -0.06 & 0.61 & 0.00 \\
\hline
\end{tabular}

${ }^{*} p<0.05 ;{ }^{* *} p<0.01 ;{ }^{* * *} p<0.001$.

A pronounced gender difference was observed in post-exercise exhaustion, with women reporting lower exhaustion scores $(M=0.98)$ than men $(M=1.28)$. Workout duration also affected post-exercise reports of exhaustion, such that, for every half hour of exercise, exhaustion decreased by 0.19 raw units or 0.35 standardized units. These effects were qualified by a significant participant gender $\mathrm{x}$ workout duration interaction, such that workout duration made little difference among men, whereas among women, increased workout duration was associated with lower post-exercise exhaustion scores (Fig. 3). A participant gender x RPE interaction also emerged, such that, for women, the increases in RPE were associated with decreases in exhaustion, whereas the opposite was true for men (Fig. 4). 


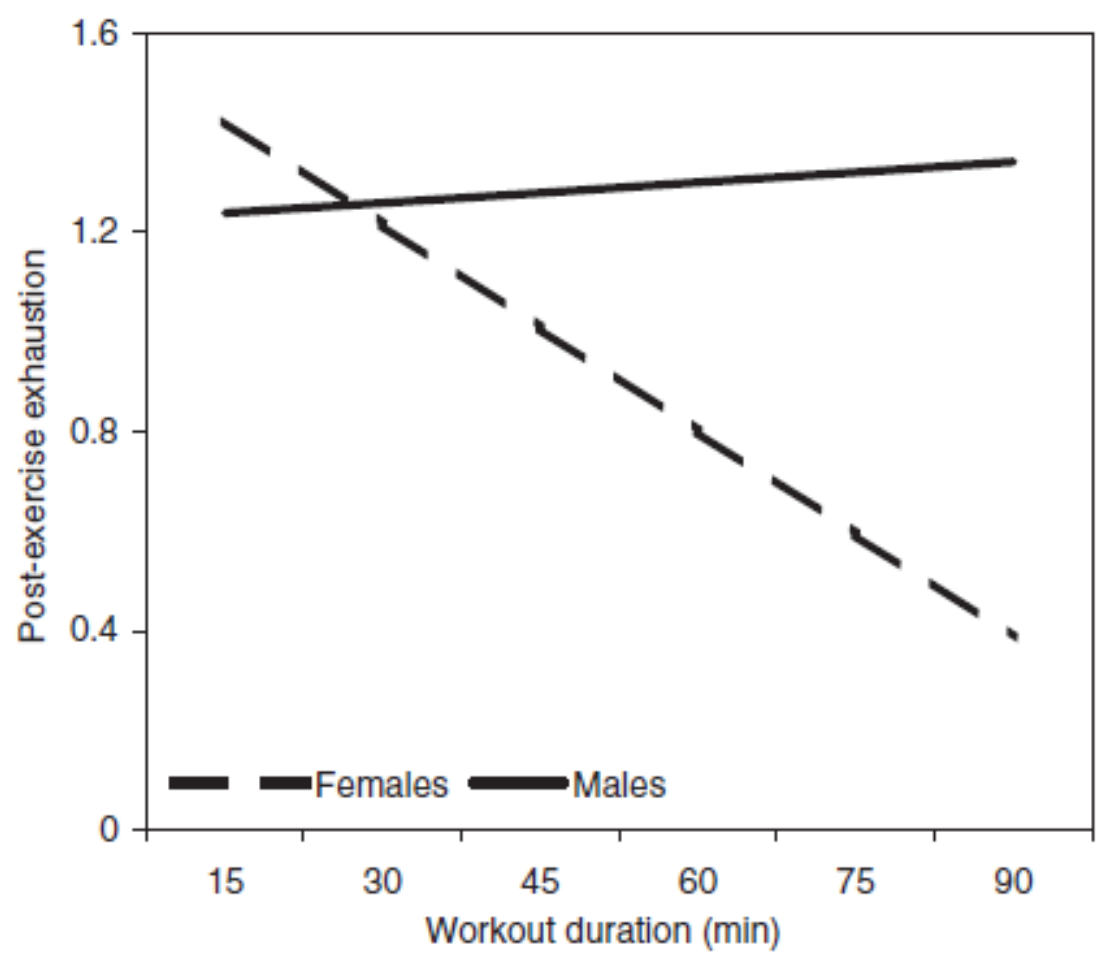

Figure 3 Post-exercise exhaustion as a function of participant gender and workout duration (min). 


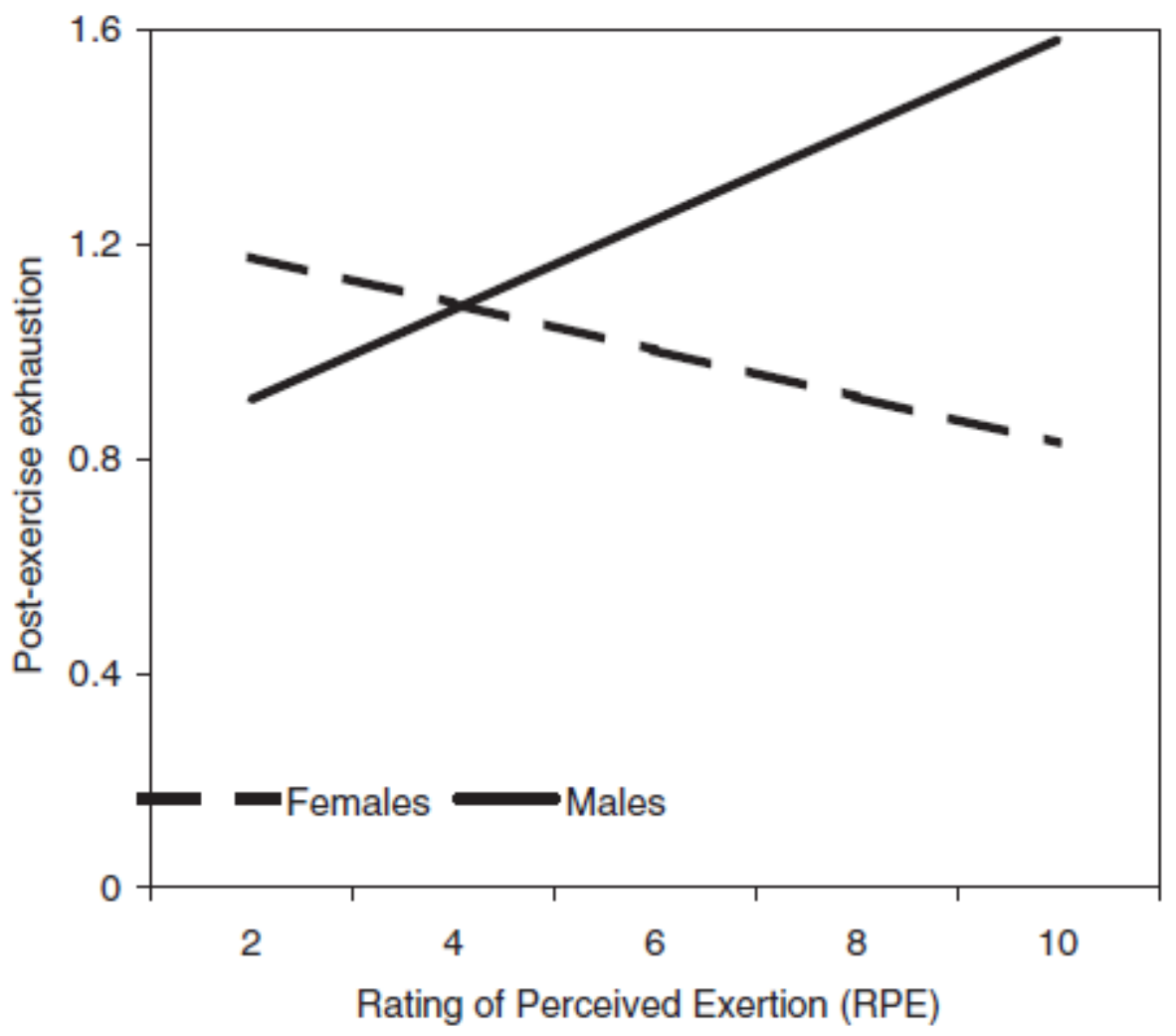

Figure 4 Post-exercise exhaustion as a function of participant gender and Rating of Perceived Exertion (RPE).

\section{Supplemental Analyses}

When the variables of participant age and its interactions with exercise type, participant gender, workout duration, and RPE were added to the models presented above, they did not explain a significant amount of variance in either post-exercise negative mood or exhaustion, $\Delta R^{2} s<0.03$, Fs $<1$, ps $>0.40$. Moreover, none of the individual age-related variables were related to either post-exercise negative mood ( $p s>0.20$ ) or exhaustion ( $p s>0.15$ ). Additionally, neither the quadratic effects of workout duration nor RPE (cf. Zervas et al., 1993) were reliable predictors of post-exercise negative mood or exhaustion when added to the model ( $p s>0.20$ ).

\section{DISCUSSION}

As predicted, we demonstrated that an acute bout of exercise led to decreased negative mood and exhaustion. These changes were observed, despite the already fairly positive mood reported by participants prior to exercise. Although it may seem counterintuitive that exhaustion would decrease after physical activity, recall that exhaustion included both physical indicators (e.g., "tired") and psychological indicators (e.g., "mentally alert"). 
Our hypotheses regarding moderators of the exercise-mood effect were also largely supported. In regard to negative mood, workout duration significantly moderated the change in negative mood due to exercise, in the predicted direction. That is, as workout duration increased, participants tended to report a greater decrease in negative mood after exercise. Our hypothesis that RPE would be positively related to post-exercise mood was partially supported. For participants who lifted weights, we observed the expected positive relationship (i.e., increased exertion led to greater decreases in negative mood). However, for participants who did cardiovascular exercise, increased RPE was related to less change in negative mood. Furthermore, as expected, women weight lifters exhibited less post-exercise negative mood than men; no such gender difference was observed among cardiovascular exercisers.

In regard to exhaustion, we observed the expected gender difference, such that women exhibited a greater decrease in exhaustion due to exercise than did men. This gender difference was exaggerated at greater workout durations and at higher levels of RPE. Therefore, our hypotheses regarding the effects of increased duration of workout and increased RPE were supported in our sample of women, but not in our sample of men. There was no evidence for a moderating effect of exercise type on change in exhaustion due to exercise.

The mechanisms by which exercise leads to improved mood are the subject of extensive research in the fields of exercise physiology, behavioral neuroscience, sports psychology, and health psychology. Currently, it is possible to forward some hypotheses about how the moderators examined in this work exert their influence on change in mood. One possible mechanism that has been suggested to account for the exercisemood relationship is that the body releases endorphins in response to exercise. These endorphins then lead to feelings of well-being, resulting in improved mood (Thoren et al., 1990). If this is indeed the mechanism by which exercise improves mood, it may shed some light on the findings that mood improved to a greater extent with longer workout durations and exertion. It may be the case that a certain threshold must be reached to result in endorphin release. This threshold may be met after a period of exercising and may only occur with a moderate to high level of exertion. [1]

The duration of workout and RPE moderating effects could also potentially be explained by the distraction (e.g., Raglin and Morgan, 1985) and mastery (Simons et al., 1985) mechanistic hypotheses. The distraction hypothesis posits that exercise improves mood indirectly by offering a distraction from life's concerns. It may be necessary to work out for a moderate to long period of time and at a moderate to difficult level of exertion to make exercise effectively distracting. The mastery hypothesis suggests that the sense of accomplishment that derives from completing a difficult activity (such as exercise) is responsible for improved mood. Indeed, past research has shown that self-efficacy is significantly related to the mood-enhancing qualities of exercise (Marquez et al., 2002) and was useful in the prediction of subsequent exercise behavior (Rodgers et al., 2002). To the extent that working out for a long period of time and at a high exertion level is more likely to result in greater feelings of accomplishment, the mastery hypothesis might also account for these moderating effects.

The finding that women benefit from greater improved mood than men might be explained by gender differences in response to any of the prior mechanisms. Alternatively, this finding can be accounted for by another proposed mechanism of 
the exercise-mood relationship, which suggests that differences in thermoregulation during exercise are associated with changes in mood (e.g., Petruzzello et al., 1993). Several exercise physiology studies have demonstrated gender differences in temperature regulation during exercise (e.g., Yousef et al., 1984; Kaciuba-Uscilko and Grucza, 2001), due to women's larger ratio of body surface to body mass and tendency to sweat less than men. Research specifically examining the linkage between gender differences in thermoregulation and their association with mood has not been done, however, and would be an interesting direction for future research. Due to the naturalistic nature of this study, one should be cautious about drawing causal inferences about our observed effects. As with all studies that do not randomize participants to condition, there may have been pre-existing differences between the groups other than those that we measured in the pretest (for instance, whether they had done another type of workout recently, fitness level/frequency of exercise, body mass index, food intake prior to exercise, sleep, time of day, etc.) that could have affected the results. Accordingly, additional research that investigates or controls for the effects of these potential moderators would be useful. For instance, future studies might include instructions for participants to record their heart rate at a specific time point into their workout. This would provide an objective measure of exertion while maintaining ecological validity. From a broader perspective, controlled laboratory studies with random assignment clearly have higher internal validity than any naturalistic design ever could. Conversely, artificial laboratory studies of the exercise-mood relationship may well suffer from a lack of external validity. Thus a combination of studies such as this naturalistic investigation as well as controlled laboratory investigations is crucial in order to maximize both internal and external validity, and obtain a full understanding of the relationship of exercise to mood enhancement. We believe that we have made a methodological advance over previous naturalistic studies - namely, that we have included a large variety of potential moderators, and have tested them in different types of exercise among both men and women. Many previous naturalistic studies have looked at only one type of exercise (i.e., resistance training or cardiovascular only; Berger et al., 1998; Bartholomew, 1999) or one population (i.e., men or women; Steptoe et al., 1993; Lane et al., 2002). Strengths of our study are that it included both men and women, engaging in both cardiovascular and weight training exercise. This allows us to directly compare moderators of the exercise-mood relationship.

Another limitation of the current study was the use of self-report. It is possible that there was a testing effect, which led people to be especially aware of their moods while exercising, therefore artificially inflating their moods. This does not seem to be likely, as moderating effects of gender, duration of workout, and RPE were detected. If testing effects were a concern, one would expect the effect to occur across all conditions.

Another potential concern is that there were demand characteristics inherent in the study. Perhaps people only reported greater mood after exercise in an attempt to be good participants. However, it is unlikely that participants would have been able to predict the differential effects expected among the moderators (i.e., gender, exercise type, duration of exercise, and RPE), making a demand characteristic explanation of the results less tenable. Also, a recent study (Berger et al., 1998) casts doubt upon these explanations. In the Berger et al. (1998) study, the researchers told half of the participants the hypothesis regarding improved mood. The other participants were supplied with a different hypothesis that did not involve the exercise-mood relationship. 
Both groups exhibited improved mood, and the difference in magnitude between groups was not significant.

One threat of conducting this type of research in a naturalistic environment is the potential for a selection bias. It is possible that exercise and mood may share a reciprocal causal relationship. For instance, individuals who start out with a positive mood may be more likely to work out for longer and at a higher level of exertion than people who begin with a more negative mood, thus confounding pre-exercise mood with the moderators of duration and RPE. However, as initial mood scores did not predict duration and exertion level, such a reciprocal relationship is not likely to account for these data. Clearly, however, the group of individuals who participated in our study (i.e., a group who chose to visit a recreation center and exercise) is different in other unmeasured ways from the population of sedentary individuals to whom we eventually wish to generalize these types of findings. Given the design of the current study, it was impossible to include sedentary participants for direct comparison to exercising participants. However, previous research findings that completely sedentary and regularly exercising participants do not differ in the degree of mood benefit after exercise (e.g., Steptoe et al., 1993) help to mitigate concerns about generalizing the current findings to sedentary populations.

One common criticism of using the POMS in this type of research is that it is heavily focused on negative mood (e.g., LeUnes and Burger, 2000) and is not directly relevant to exercise behavior. Therefore, replicating the current study while using a mood scale that included more positive affect may be warranted. A measure we are currently utilizing to address this concern in some ongoing research in this area is the Physical Activity Affect Scale (PAAS; Lox et al., 2000), which assesses exercise-induced feeling states such as positive affect, negative affect, tranquility, and physical exhaustion. The PAAS shows adequate internal consistency and discriminant validity among the factors (Lox et al., 2000), is balanced between positive and negative affective states, and is specifically geared to testing exercise-induced mood changes.

An exciting additional direction for future research regarding the exercise-mood relationship is to test whether immediate positive responses to exercise behavior are associated with the maintenance of exercise behavior over the longer term. Despite clear indications that acute exercise positively influences mood, several studies have failed to find an association between the effects of exercise on mood and level of previous exercise behavior. For example, although a few studies have noted that previously active individuals report more positive affect after acute exercise (e.g., Boutcher and Landers, 1988; Petruzzello et al., 2001), other studies do not support this conclusion (Roth, 1989; Choi et al., 1993; Steptoe et al., 1993; Hansen et al., 2001). Further, there are no studies of which we are aware that demonstrate that positive mood changes in response to exercise are predictive of future exercise behavior. If we are to eventually capitalize on changes in mood encouraging future exercise behavior, the validity of this relationship must first be demonstrated empirically.

The findings of the current study have implications for the design of programs intended to increase exercise behavior. If people who are starting an exercise program benefit from mood enhancement during the adoption phase, it is likely that they will be more successful in maintaining a regular exercise routine. While the results of the current study need to be replicated, some tentative suggestions regarding intervention foci can be made. We found no difference in mood improvement due to exercise 
type, suggesting that interventions with a mood focus may not need to differentiate between cardiovascular exercise and weight training. Rather, interventions might encourage working out at a moderate to high level of exertion, and for a moderate to long period of time. Such interventions focusing on improved mood may be particularly effective with women, as they showed the greatest overall mood enhancement.

In addition to these general recommendations, preliminary prescriptions for exercise can be outlined for exercise within each gender. Given our findings, it appears that women should be encouraged to participate in weight training, at a moderate to high exertion level, and that these training sessions should be of moderate or long duration (i.e., an hour or more). These conditions should result in the greatest mood enhancement after exercise among women. It is more difficult to outline specific recommendations for men. In general, men should be encouraged to work out at a low to moderate level of exertion, whether engaging in cardiovascular or weight training. As men experienced less mood enhancement overall, other strategies for promoting exercise in men may be more effective.

\section{CONCLUSION}

This study demonstrated that an acute bout of exercise can lead to improved mood outside of a laboratory environment. The results are encouraging, as cardiovascular exercise and weight training were found to be equally effective in improving mood.

Future research to further identify the effect of moderators of the exercise-mood relationship, and to establish whether participants who experience improved mood exhibit greater exercise maintenance, should help to inform the design of exercise promotion programs. 


\section{FOOTNOTES}

1. The literature on changes in plasma endorphin levels due to exercise and resultant mood changes is equivocal. Generally, beta endorphin levels have been found to increase during moderate to intense (i.e., 60-80\% of Maximum Heart Rate; MHR) cardiovascular exercise, but not in lower intensity cardiovascular exercise (i.e., 40-60\% of MHR; e.g., Kraemer et al., 1989; Goldfarb et al., 1998). Other studies have found that cardiovascular workouts at $80 \%$ of MHR led to decreases of beta endorphin (Kraemer et al., 1990). Typically, no gender differences in beta endorphin levels at pre- or post-exercise have been observed and the relationship between mood and beta endorphin levels has been similar for men and women. However, Kraemer et al. (1990) found that, while no relationship between beta endorphin levels and mood emerged in women, decreased beta endorphin led to improved mood in men. More commonly, researchers have reported a positive relationship between beta endorphin levels and enhanced mood resulting from cardiovascular exercise (e.g., Harte et al., 1995). In contrast to cardiovascular exercise, beta endorphin levels seem most commonly to decrease in response to resistance exercise (e.g., McGowan et al., 1993; Pierce et al., 1994). McGowan et al. (1993) observed no relationship between beta endorphin level and mood. The diverse findings in this area may result from difficulties in measuring endorphin levels. The endorphin hypothesis posits that endorphin levels in the brain should be positively related to mood. However, measuring CNS endorphin levels is a highly invasive procedure that would lead to physical and psychological changes itself (Yeung, 1996). The dominant approach to this problem has been to measure plasma, or peripheral, endorphin levels, which is much less invasive. Unfortunately, it is unclear whether plasma endorphin levels correlate well with CNS levels, making work in this area difficult to interpret. 


\section{REFERENCES}

Bartholomew, J.B. (1999). The effect of resistance exercise on manipulated preexercise mood states for male exercisers. Journal of Sport \& Exercise Psychology, 21(1), 39-51.

Berger, B.G., Owen, D.R., Motl, R.W. and Parks, L. (1998). Relationship between expectancy of psychological benefits and mood alteration in joggers. International Journal of Sports Psychology, 29(1), 1-16.

Blair, S.N., Kampert, J.B., Kohl, H.W., Barlow, C.E., Macera, C.A., Paffenbarger, R.S., Jr. and Gibbons, L.W. (1996). Influences of cardiorespiratory fitness and other precursors of cardiovascular disease and all-cause mortality in men and women. Journal of the American Medical Association, 276(3), 205-210.

Bouchard, C., Shephard, R.J. and Stephens, T., Eds. (1994). Physical Activity, Fitness, and Health: International Proceedings and Consensus Statement. Hum. Kinet, Champaign, IL.

Boutcher, S.H. and Landers, D.M. (1988). The effects of vigorous exercise on anxiety, heart rate, and alpha activity of runners and nonrunners. Psychophysiology, 25(6), 696-702.

Carpenter, C.L., Ross, R.K., Paganini-Hill, A. and Bernstein, L. (1999). Lifetime exercise activity and breast cancer risk among post-menopausal women. British Journal of Cancer, 80, 18521858.

Centers for Disease Control (CDC), (2001). Physical activity trends - United States, 1990-1998. Atlanta. Available at:

http://www.phppo.cdc.gov/cdcrecommends/showarticle.asp?a_artid=1298++++\&TopNum=50\& CallPg=Adv

Choi, P.Y., Van Horn, J.D., Picker, D.E. and Roberts, H.I. (1993). Mood changes in women after an aerobics class: a preliminary study. Health Care for Women International, 14(2), 167-177.

Cohen, J. (1988). Statistical Power Analysis for the Behavioral Sciences, 2nd Edn. Erlbaum, Hillsdale, NJ. Dyer, J.B. and Crouch, J.G. (1988). Effects of running and other activities on moods. Perceptual and Motor Skills, 67, 43-50.

Farrell, P.A., Gustafson, A.B., Morgan, W.P. and Pert, C.B. (1987). Enkephalins, catecholamines, and psychological mood alterations: effects of prolonged exercise. Medicine \& Science in Sports \& Exercise, 19, 347-353.

Felts, W.M., Crouse, S. and Brunetz, M. (1988). Influence of aerobic fitness on ratings of perceived exertion during light to moderate exercise. Perceptual \& Motor Skills, 67, 671-676.

Gauvin, L. and Spence, J.C. (1996). Physical activity and psychological well-being: knowledge base, current issues, and caveats. Nutrition Reviews, 54(4, Pt. 2), S53-S65.

Goldfarb, A.H., Jamurtas, A.Z., Kamimori, G.H., Hegde, S., Otterstetter, R. and Brown, D.A. (1998). Gender effect on beta-endorphin response to exercise. Medicine and Science in Sports and Exercise, 30, 1672-1676. 
Hansen, C.J., Moses, K. and Gardner, C. (1997). The effects of time-incremented running on mood states of college atheletes. Psi Chi Journal of Undergraduate Research, 2, 133-139.

Hansen, C.J., Stevens, L.C. and Coast, J. (2001). Exercise duration and mood state: how much is enough to feel better? Health Psychology, 20, 267-275.

Kaciuba-Uscilko, H. and Grucza, R. (2001). Gender differences in thermoregulation. Current Opinion in Clinical Nutrition and Metabolic Care, 4, 533-536.

Kraemer, R.R., Blair, S., Kraemer, G.R. and Castracane, V.D. (1989). Effects of treadmill running on plasma beta-endorphin, corticotropin, and cortisol levels in male and female $10 \mathrm{~K}$ runners. European Journal of Applied Physiology and Occupational Physiology, 58, 845-851.

Kraemer, R.R., Dzewaltowski, D.A., Blair, M.S., Rinehardt, K.F. and Castracane, V.D. (1990). Mood alteration from treadmill running and its relationship to beta-endorphin, corticotropin, and growth hormone. The Journal of Sports Medicine and Physical Fitness, 30, 241-246.

Lane, A.M., Crone-Grant, D. and Lane, H. (2002). Mood changes following exercise. Perceptual \& Motor Skills, 94, 732-734.

LeUnes, A. and Burger, J. (2000). Profile of mood states research in sport and exercise psychology: past, present, and future. Journal of Applied Sport Psychology, 12(1), 5-15.

Lox, C.L., Jackson, S., Tuholski, S.W., Wasley, D. and Treasure, D.C. (2000). Revisiting the measurement of exercise-induced feeling states: The Physical Activity Affect Scale (PAAS). Measurement in Physical Education and Exercise Science, 4, 79-95.

Marquez, D.X., Jerome, G.J., McAuley, E., Snook, E.M. and Canaklisova, S. (2002). Selfefficacy manipulation and state anxiety responses to exercise in low active women. Psychology and Health, 17, 783-791.

Mather, A.S., Rodriguez, C., Guthrie, M.F., McHarg, A.M., Reid, I.C. and McMurdo, M.E.T. (2002). Effects of exercise on depressive symptoms in older adults with poorly responsive depressive disorder: randomised controlled trial. British Journal of Psychiatry, 180, 411-415.

McGowan, R.W., Pierce, E.F., Eastman, N., Tripathi, H.L., Dewey, T. and Olson, K. (1993). Betaendorphins and mood states during resistance exercise. Perceptual and Motor Skills, 76, 376-378.

McGowan, R.W., Pierce, E.F. and Jordan, D. (1991). Mood alterations with a single bout of physical activity. Perceptual \& Motor Skills, 72, 1203-1209.

McNair, D., Lorr, M. and Droppleman, L.F. (1971). Manual for the Profile of Mood States. Educational and Industrial Testing Service, San Diego.

Merns, K. (1995). Mood and self-esteem enhancement in different exercise modes. Dissertation Abstracts International: Section B: The Sciences and Engineering, 55, 5571.

Petruzzello, S.J., Hall, E.E. and Ekkekakis, P. (2001). Regional brain activation as a biological marker of affective responsivity to acute exercise: influence on fitness. Psychophysiology, 38(1), 99-106. 
Petruzzello, S.J., Landers, D.M. and Salazar, W. (1993). Exercise and anxiety reduction: examination of temperature as an explanation for affective change. Journal of Sport \& Exercise Psychology, 15, 63-76.

Pierce, E.F., Eastman, N.W., McGowan, R.W., Tripathi, H., Dewey, W.L. and Olson, K.G. (1994). Resistance exercise decreases beta-endorphin immunoreactivity. British Journal of Sports Medicine, 28, 164-166.

Pronk, N.P., Crouse, S.F. and Rohack, J.J. (1994). Maximal exercise and acute mood response in women. Physiology \& Behavior, 57, 1-4.

Raglin, J.S. and Morgan, W.P. (1985). Influence of vigorous exercise on mood state. Behavioral Therapy, 8, 179-183.

Raglin, J.S., Turner, P.E. and Eksten, F. (1993). State anxiety and blood pressure following 30 minutes of leg ergometry or weight training. Medicine \& Science in Sports \& Exercise, 25, 10441048.

Rodgers, W.M., Hall, C.R., Blanchard, C.M., McAuley, E. and Munroe, K.J. (2002). Task and scheduling self-efficacy as predictors of exercise behavior. Psychology and Health, 17, 405416.

Roth, D.L. (1989). Acute emotional and psychophysical effects of aerobic exercise. Psychophysiology, 26, 593-602.

Sallis, J.F., Haskell, W.L., Fortmann, S., Vranizan, K.M., Taylor, C.B. and Solomon, D.S. (1986). Predictors of adoption and maintenance of physical activity in a community sample. Preventive Medicine, 15, 331-341.

Salmon, P. (2001). Effects of physical exercise on anxiety, depression, and sensitivity to stress: a unifying theory. Clinical Psychology Review, 21, 33-61.

Sherwood, N.E. and Jeffery, R.W. (2000). The behavioral determinants of exercise: implications for physical activity interventions. Annual Review of Nutrition, 20, 21-44.

Simons, A., McGowan, C.R., Epstein, L.H., Kupfer, D.J. and Robertson, R.J. (1985). Exercise as a treatment for depression: an update. Clinical Psychology Review, 5, 553-568.

Steptoe, A., Kearsley, N. and Walters, N. (1993). Acute mood responses to maximal and submaximal exercise in active and inactive men. Psychology \& Health, 8, 89-99.

Thoren, P., Floras, F.S., Hoffman, P. and Seals, D.R. (1990). Endorphins and exercise: physiological mechanisms and clinical implications. Medicine and Science in Sports and Exercise, 22, 417-428.

Tiggeman, M. and Williamson, S. (2000). The effect of exercise on body satisfaction and selfesteem as a function of gender and age. Sex Roles, 43, 119-127.

Twamley, E.W. (2000). Gender, exercise motives, and exercise behavior. Dissertation Abstracts International: Section B: The Sciences \& Engineering, 61, 1099. 
U.S. Department of Health and Human Services (USDHHS), (2000). Healthy People 2010. (Conference Edition in two Volumes). Washington DC. Available at:

http://www.health.gov/healthypeople/.

Yousef, M.K., Dill, D.B., Vitez, T.S., Hillyard, S.D. and Goldman, A.S. (1984). Thermoregulatory responses to desert heat: age, race, and sex. Journal of Gerontology, 39, 406-414.

Yueng, R.R. (1996). The acute effects of exercise on mood state. Journal of Psychosomatic Research, 40, 123-141.

Zervas, Y., Ekkekakis, P., Emmanuel, C., Psychoudaki, M. and Kakkos, V. (1993). The acute effects of increasing levels of aerobic exercise intensity on mood states. In: Serpa, S., Alves, J., Ferreira, V. and Paulo-Brito, A. (Eds.). Proceedings of the 8th World Congress of Sport Psychology. Lisbon, Portugal. 